\title{
The Role and Application of Salivary Exosomes in Malignant Neoplasms
}

\author{
Yongqin Deng \\ Yujie Cao \\ Liuqian Wang \\ Dong Ye $\mathbb{D}$
}

Department of OtorhinolaryngologyHead and Neck Surgery, Lihuili Hospital of Ningbo University, Ningbo, Zhejiang, 315040, People's Republic of China
Correspondence: Dong Ye

Department of Otorhinolaryngology-Head and Neck Surgery, Lihuili Hospital of Ningbo

University, Ningbo, Zhejiang, 315040,

People's Republic of China

$\mathrm{Tel}+86-138$ I986 I2I3

Fax +86-574-8739 2232

Email yedong518@sina.com
Abstract: The study of salivary exosomes in malignant neoplasms has attracted widespread attention in the clinical setting. Although a variety of diagnostic and treatment approaches have been proposed, there are some limitations to their application. In recent years, the role of salivary exosomes in cancer has been increasingly studied. Salivary exosomes not only renew and regulate the biological behavior of tumor cells, such as malignant proliferation, migration, and invasion, but they also serve as ideal markers for early diagnosis of diseases and may represent an effective therapeutic target. This article reviews the current research on salivary exosomes in malignant neoplasms.

Keywords: liquid biopsy, exosome, malignant neoplasm, diagnosis, treatment

\section{Introduction}

An epidemiological survey of malignancies estimated that about 14 million new cancer cases worldwide were diagnosed in 2012 and this number will rise to 24 million by $2035 .^{1}$ Of these, the number of new cancer cases in the elderly is expected to double, which may increase the global economic burden of cancer in terms of the demand for clinical services. At present, the early diagnosis of malignant tumors is difficult, and common treatment mainly includes surgery, chemotherapy, radiotherapy, and targeted therapy, however, the impact of treatment on outcomes is still not ideal. Further, treatment may also damage normal tissues and lead to serious complications. Therefore, finding new and effective approaches for diagnosis and treatment and exploring the mechanisms that affect the occurrence and development of tumors have always been the focus of ongoing research.

Saliva is a mixture of water, electrolytes, enzymes, and other elements. ${ }^{2}$ Molecules in the saliva can be exchanged with blood through capillaries in the glands, hence, they share similar bioactive substances. ${ }^{3}$ In addition, as a sampling material, saliva has a widely accessible and is easy to collect. Compared with clinical diagnoses using other body fluids, such as cerebrospinal fluid, thoracic effusion, and blood, sampling of saliva has the advantages of being safe, noninvasive, of low cost, and convenient.

Exosomes are nanosomes with a diameter of about 50 to $140 \mathrm{~nm}$ that are actively secreted by a variety of cells (macrophages, tumor cells, and mast cells) in the body, with an outer shell composed of a lipid bilayer structure. ${ }^{4}$ Exosomes were first identified by Johnstone et al $^{5}$ in the reticulocytes of mammals, and were initially only regarded as "waste" at that time; hence, they were not given much attention. Recent studies have found that exosomes are spherical, their cores are 
composed of hydrophilic structures, and vesicles are rich in proteins, lipids, and genetic material. ${ }^{6}$ Exosomes are widely present in bodily fluids such as saliva, blood, cerebrospinal fluid, and urine, and play a role in cell communication or as mediators of cell communication through the release of bioactive substances contained in their vesicles. ${ }^{7-9}$

Studies have confirmed that bioactive substances in salivary exosomes are involved in physiological or pathological processes of the body, ${ }^{3}$ and are closely related to head and neck malignancies such as laryngeal, hypopharyngeal, thyroid, oral, nasopharyngeal cancers, and many other systemic tumors. It is relatively easy to isolate salivary exosomes. Compared with other body fluids, salivary exosomes have unique advantages, high stability, and strong tissue targeting capabilities. A close relationship between exosomes and malignancies has been demonstrated, allowing them to regulate tumor growth, invasion, and metastasis, and to monitor the prognosis of malignancies. Due to their structural characteristics, salivary exosomes present their own structural characteristics, which are not easily degraded by enzymes in the body. When transmitting messages, the integrity and biological function of vesicles are maintained, which is conducive to their extraction as experimental samples and detection of diseases. ${ }^{10}$ Studies have shown that the majority of RNA in salivary exosomes are microRNA (miRNA), whose content and structure vary in tumor patients. Therefore, salivary exosomes can also be used as a tool for early diagnosis of tumors. ${ }^{11}$ In addition, salivary exosomes can be used as carriers of anti-tumor drugs, and may play a role in the communication between tumor cells to reshape the tumor microenvironment, thus becoming an effective target for tumor therapy. Fu et $\mathrm{al}^{12}$ extracted human $\mathrm{T}$ cells and evaluated their activity on tumors in vitro and in vivo in nude mice. The authors found that $\mathrm{T}$ cells expressing chimeric antigen receptor (CAR-T) secreted surface exosomes carrying CAR that could bind tumor cells, inhibit tumor growth, and kill tumors. Exosomes with reversible fusion and fission capabilities have been developed to control the delivery of drug-carrying exosomes during $\mathrm{Fe} 2+$ and $\mathrm{Fe} 3+$ interredox processes. ${ }^{13}$ Engineered exosomes can be re-programmed for the release of exosomaltargeted drugs under photothermal induction, suggesting that exosomes can be used for precision oncology therapy. ${ }^{14}$ The study of salivary exosomes and their association with malignant tumors have recently attracted much attention, and the findings provide broad prospects for noninvasive detection of tumors.

\section{Overview of Salivary Exosomes Composition of Salivary Exosomes}

Salivary exosomes can be secreted by salivary glands or blood. They are round vesicles in shape and contain shared proteins, such as heat shock protein 70 (HSP70) and tetraminohydrin. These proteins, which can also be found in exosomes from other sources, are closely related to calcium transport, signal transduction, protein processing, and transportation, and also contain specific proteins, such as AQP5 or aquaporin 5, involved in salivary water regulation. ${ }^{10,15}$ Some proteins co-exist in salivary exosomes and saliva, and are highly expressed in the former, indicating that these proteins may originate from salivary exosomes. ${ }^{3}$ In addition to proteins, salivary exosomes also contain bioactive molecules such as mRNA, miRNA and cytokines. Studies have shown that mouse proteins can be expressed in human mast cells by adding mouse mast cell exosomes to human mast cells, and mast cell-derived exosomes can exchange RNA through exosomes. Exosome RNAs that can travel between cells are called "esRNAs", and may involve miRNAs, a class of noncoding mRNAs with sizes that vary from 19 to 25 nucleotides in length and that mediate cell-to-cell communication in the microenvironment, as well as play an important role in tumor diagnosis. ${ }^{16-18}$

\section{Separation of Salivary Exosomes}

Based on chemical and physical properties, a variety of separation methods can be used to isolate salivary exosomes, including membrane affinity ultracentrifugation, tangential flow filtration, field flow fractionation, sucrose gradient centrifugation, ExoQuick extraction, and size exclusion chromatography. To separate components of saliva secreted by the body there are two types of separation methods. The first is a form of ultracentrifugation and the other involves chemical precipitation. ${ }^{19}$ Both methods present some limitations: the ultracentrifugation method is simple to perform, but is time consuming and produces exosomes of low purity. Sedimentation can obtain complete exosomes and increases their recovery, but it is only suitable for specific scenarios. ${ }^{20,21}$ Due to the fact that exosomes are easily damaged and contaminated, and viscous substances are obtained following ultracentrifugation, the separation of salivary exosomes is still difficult to 
achieve at present. A newly developed ultra-fast separation and purification system (EXODUS) presents the advantages of low cost, high stability, and reproducibility, and improves the purity, efficiency, and yield of exosomes. This improved exosome separation technology will allow to discover more exosomal diagnostic disease markers, to be exploited for early disease screening. ${ }^{22}$

\section{Function of Salivary Exosomes}

Studies have shown that salivary exosomes contain tissue factor (TF) and participate in the initial stages of coagulation in the body, shorten the coagulation time, and play a role in hemostasis. ${ }^{23}$ Minor variations in salivary exosome content are diagnostic for a variety of diseases, such as autoimmune disease, ${ }^{24}$ periodontitis, ${ }^{25}$ and oral lichens planus. ${ }^{26}$ In tumors, molecules in saliva can be exchanged with blood through capillaries in glands, and about $80 \%$ of proteins coexist with exosomes in saliva and serum.

Studies have shown that exosomes can be used as diagnostic markers in multiple tumors. ${ }^{27,28}$ Salivary exosomes are rich in angiogenic proteins that deliver growth factors and stimulate endothelial cells, thereby promoting angiogenesis in tumor tissues. ${ }^{29}$ In addition, salivary exosomes can adapt to and participate in host humoral immunity, enhance immune resistance of tumor cells, evade the immune surveillance of NK cells, and promote tumor immune escape. ${ }^{21,22}$ Salivary exosomes also have the ability to deliver P-glycoprotein (P-gp), which mediates mechanisms of drug-resistant metastasis, and enhances tumor resistance. ${ }^{30}$ Thus, salivary exosomes create favorable conditions for tumor migration and invasion. ${ }^{31,32}$ At present, salivary exosomes can also assist in the delivery of anti-tumor drugs or interfere with tumor growth to exert their an anti-tumor role. ${ }^{33}$

\section{Role of Salivary Exosomes in Diagnosis and Prognosis Evaluation of Malignant Tumors}

Salivary exosomes are suitable for tumor fluid biopsies, as they are rich in a variety of active substances and can be used for early diagnosis and prognosis evaluation of tumors. ${ }^{34}$ Salivary exosomes containing miRNA and circulating tumor genes (ctDNA) can be used as biomarkers for cancer screening. ${ }^{35} \mathrm{~A}$ head and neck squamous cell carcinoma (HNSCC) study found that using ctDNA as a marker extracted from oral saliva, the early tumor positive diagnostic rate was $100 \%$, which was significantly higher than blood tests, and also presents higher sensitivity for diagnosis of cancers of the distal oral cavity (oropharynx, larynx, and hypopharynx). ${ }^{36}$ Studies including 5 groups of HNSCC and healthy controls showed that miR-486-5p or miR-486-3p and miR-10b-5p were significantly upregulated in patients with HNSCC, and miR-486-5p was also significantly altered in early lesions (stage I) and thus could be applied as a biomarker for early diagnosis of HNSCC. ${ }^{37}$

Liu et al compared salivary exosomes of oral cancer patients and healthy controls by quantitative real-time polymerase chain reaction (qRT-PCR), and found that the increased expression of miR-31 could promote higher expression of miR-29a-3p mediated by exosomes by regulating the SOCS1/STAT6 signaling pathway in macrophages. Further, the expression of miR-125a and miR200a was decreased, and a reduction of miRNA was observed after tumor resection, which can be used to monitor tumor prognosis. ${ }^{38,39}$

Lin et al identified higher levels of GOLM1-NAA35 RNA, a chimeric RNA (seG-NchiRNA) secreted by salivary exosomes in mice and humans in early- and advanced-stage esophageal squamous cell carcinomas (ESCC) compared with the normal control group. ${ }^{40}$ Nano-particle tracking analysis showed that the level of seG-NchiRNA in ESCC patients was 2.1 times higher than that in the normal control group, and the changes in the level of seG-NchiRNA were correlated with changes before and after ESCC surgery, which provide a scientific and effective rationale for monitoring the prognosis of ESCC patients.

Zhang et $\mathrm{al}^{41}$ found that the changes in the expression of various mRNAs and carbonic anhydrase 6 (CA6) in salivary exosomes of breast cancer patients were more marked than those in healthy controls, which could be used for the early diagnosis of breast cancer. Lau et al established an in vitro model of breast cancer and found through microarray analysis that exosomes derived from breast cancer cells could process the transcription of salivary exosomes, thereby changing the content of the salivary exosome. ${ }^{42}$

In a mouse model, exosomes secreted by pancreatic cancer cells were able to regulate the transcription of genes in the saliva, and further, pancreatic cancer-specific transcriptional genes were identified as having increased expression in salivary exosomes, indicating the presence of markers of pancreatic cancer cells in salivary exosomes. $^{43}$ 
Sun et al used quantitative methods to compare the salivary exosome proteomics profile of normal subjects and patients with lung cancer, and confirmed that four proteins (BPIFA1, CRNN, MUC5B, and IQGAP) derived from lung tissue cells and could be used for non-invasive detection of lung cancer, and further, approximately $80 \%$ of the proteins in salivary exosomes and serum exosomes were shared. These findings indicated bi-directional communication of information and correlation of composition between salivary exosomes and distal tumors. ${ }^{44,45}$ To further confirm this relationship, a tumor transplantation model of nude lung cancer mice was established. H460 lung cancer cells were engineered to stably express HCD63-GFP, and were then injected into the thoracic cavity of nude mice. Human GAPDH mRNA was detected in the HCD63+ saliva exosomes of experimental nude mice. These results indicated that specific mRNAs in salivary exosomes could be used to identify tumors and have the potential to be biomarkers for tumor diagnosis. ${ }^{15}$

\section{Role of Salivary Exosomes in the Treatment of Malignant Tumors}

Due to its own structural characteristics, exosomes become "cargo" nanocarriers with unique advantages, including the inclusion and protection of its specific contents, low rejection rates, and drug resistance, permeability of the blood-brain barrier, high efficiency of drug delivery, and maintenance of drug activity. Therefore, exosomes have the potential for wide application in disease treatment. ${ }^{46}$ Paclitaxel (PTX) presents unique anti-tumor activity by inhibiting cell microtubule mitosis. Exosome inclusion of PTX can improve drug stability and blood drug concentration, and enhances the inhibitory effects on cancer cell proliferation. ${ }^{47,48}$ Ma et al found that exosomes secreted by breast ductal cancer cells carrying antitumor drugs such as methotrexate can help inhibit reverse the proliferative activity of CD133+ tumor stem cells, indicating that exosomes can enhance the efficacy of antitumor drugs and kill tumor cells. ${ }^{49}$ Exosomes carrying antioxidant drugs can reach neuron cells through intranasal administration and provide a protective effect on neuron activity. ${ }^{50}$ Exosomes carrying IFN- $\gamma$ can increase the secretion of IFN- $\gamma$ by NK cells, which has a potential role in the treatment of tumor cells. ${ }^{51}$

Salivary exosomes can also be used as targets for tumor therapy. Baroni et al found that salivary exosomes carrying miR-9 are significantly increased in oral squamous cell carcinoma (OSCC) and could promote tumor growth by inducing fibroblast-like characteristics of breast cancer cells, while the introduction of LNA-9, an inhibitor of miR-9, could reduce tumor metastasis and invasion. ${ }^{52,53}$ Li et al demonstrated that $\gamma \delta \mathrm{T}$ cell extracellular vesicles $(\gamma \delta \mathrm{T}$ DES) rich in miR-138 can increase the number of G1 stage tumor cells in nude mice, inhibit the biological activity of CAL-27 cells, slow down the growth rate, and inhibit tumor formation. ${ }^{54}$ In addition, knockdown of miR$133 \mathrm{~b}-3 \mathrm{p}$ in saliva exosomes reduces the expansion of epithelial progenitor cells, providing a new rationale for tumor therapy. ${ }^{55}$

Studies have shown that exosomes released by cancer cells can inhibit the activation and proliferation of CD8+T cells, promote the regulation of $\mathrm{T}$ cells, and play an immunosuppressive role. Immunotherapy of exosomes plays an important role in human tumors, and lays the foundation for the research and development of tumor immunotherapy vaccines. ${ }^{56,57}$

\section{Mechanism of Action of Salivary Exosomes in Malignant Tumors Promotion of Tumor Progression}

Tumorigenesis is a dynamic and complex process. ${ }^{58}$ Salivary exosomes can influence the progression of tumor cells through signaling molecules, and are closely associated with multiple biological processes of malignant tumors, thereby mediating the malignant progression of tumor cells. ${ }^{59}$

\section{Salivary Exosomes and Tumor Proliferation}

About $90 \%$ of head and neck malignancies are HNSCC, which are common systemic malignancies. Oral cancer is a common subtype of head and neck malignancies and is increasingly affecting younger individuals in recent years, likely due to an association with viral infections and personal lifestyle habits. ${ }^{60}$ Distant metastasis may occur early in the disease course and the prognosis is poor. When most patients are diagnosed clinically, they have already developed to the advanced stage and have missed the optimal treatment time. Therefore, it is extremely important to explore early and efficient detection methods. Gai et $\mathrm{al}^{61}$ showed that salivary extracellular vesicle-associated miRNA can affect OSCC, mainly through miRNA ErbB signaling pathways, while targeted regulation of CD44 can promote tumor cell proliferation and invasion. In addition, miR-512-3p and miR-412-3p can target multiple genes in the TGF $\beta$ signaling pathway (for example, 
TGF $\beta R 2$ ) to decrease the regulation ability of suppressor genes. The specific upregulation of miR-302b-3p and miR-517b-3p expression was confirmed by qRTPCR in OSCC patients. Hypoxia-induced OSCC cellderived exosomes stimulate miR-21 expression by inducing HIF- $1 \alpha$ and HIF-2 $\alpha$, thereby promoting tumor growth. ${ }^{62} \mathrm{He}$ et $\mathrm{al}^{63}$ up-regulated levels of salivary exosome miR-24-3p, which then directly acted on the transcription initiation of target genes in cancer cells, and thereby inhibited the expression of PER1 and improved the growth rate and colony-formation efficiency of OSCC cells. Wang et $\mathrm{al}^{64}$ extracted exosomes from salivary glands derived from adenoid cystic cancer cells using a commercial kit. These extracted exosomes were found to enhance tumor cell invasion and metastasis by regulating kinase ERK1/2 phosphorylation, the MAPK signaling pathway, and promoted fibroblast morphogenesis through the NGF-NTRK1 signaling pathway, thus promoting tumorgenicity. Zhang et $\mathrm{al}^{65}$ found that the salivary exosome miR-512-3p in patients with TSCC promoted the proliferation of tumor cells by down-regulating GAS2. Salivary exosomes could also reduce the cytotoxicity of NK cells by down-regulating the expression of CD69 and NKG2D, which resulted in an immune escape mechanism, which promoted tumor proliferation. ${ }^{66}$

\section{Salivary Exosomes and Tumor Metastasis}

Chen et $\mathrm{al}^{67}$ identified in Epstein-Barr virus-positive nasopharyngeal carcinoma that exosomes could induce lymphatic ducts and tube formation, thereby promoting nasopharyngeal lymphatic metastasis. Latent membrane protein 1 (LMP1) was identified in the saliva of exosomes. ${ }^{68}$ LMP1 protein can up-regulate the expression of NTRK2 through NF-kB to activate Akt and ERK signal transduction and enhance the hypoxic tolerance and metastasis of tumor cells. ${ }^{69}$ EB-LMP1 can also activate the IRF7 signaling pathway, increase the expression of A3S, cause mtDNA hypermutation, and alter the expression of miRNA, and ultimately promotes tumor cell invasion and distant metastasis. $^{70}$

\section{Tumor Inhibition}

Cancer-derived inhibitory salivary exosomes exert an anti-tumor role by influencing angiogenesis and regulating stem cell properties. Gonzalez-Begne et al identified angiopoietin-like protein 1 (ANGPTL1) in salivary exosomes, which is widely present in human tissues, and found that it regulates miR-630 promoter activity and inhibits zinc finger protein (SLUG) expression by down-regulating the ERK/SP1 signaling pathway. SLUG is overexpressed in a variety of malignant tumors and is related to the stem cell properties and drug resistance of tumor cells, and thus has an inhibitory effect on the metastasis, proliferation, and progression of cancer. ${ }^{71-73}$ Studies have shown that miRNA16, an carried by exosome secreted by mesenchymal stem cells from mice, reduced the expression of vascular endothelial growth factor (VEGF) in $\mathrm{T} 1$ cells in a dose-dependent manner, reduced tumor proliferation and migration, and altered tumor progression. ${ }^{74}$ VPS4A is tumor suppressor that mediates miRNAs, such as miR-122-5p, miR-29b-3p secretion, via exosomes derived from liver cancer cells. Through the PI3K/Akt signaling pathway in liver cancer cells, VPS4A regulates the uptake of tumor exosome miRNAs, thereby inhibiting tumor progression. ${ }^{75}$

Bioactive substances of salivary exosomes play different roles in different malignant tumors. The bioactive substances and the mechanism of salivary exosomes in different types of malignant tumors are shown in Table 1.

\section{Summary and Prospects}

As a non-invasive material, salivary exosomes have the advantages of relatively stable sampling and convenient storage, which are helpful for the diagnosis and prognosis of a variety of tumors, and thus, represent a new direction in the diagnosis and treatment of malignant tumors. Several studies have examined the role of exosomes in diagnosis, treatment and mechanisms underlying of salivary exosome-mediated metastasis in malignant tumors. However, the mechanisms involved in the regulation of salivary exocrine exosomes by the body are currently not clear; thus, exploring separation technologies, improving precision medical treatment by understanding the properties of saliva secreted by body and regulatory mechanism controlling malignant tumors should be further clarified. Salivary exosomal carriers of antitumor drugs can be engineered for clinical use, as their immunosurveillance capabilities and potential application for immunotherapy treatment show great potential. Extracting salivary exosomes and designing engineering exosomes for clinical application in precise tumor treatment will represent the research direction in the future. 
Table I Mechanism of Action of Salivary Exosomes in Malignant Tumors

\begin{tabular}{|l|l|l|l|l|}
\hline Disease & Bioactive Substance & Pathways & Effect & Reference \\
\hline OSCC & miR-5I2-3p & $\begin{array}{l}\text { ErbB and CD44 } \\
\text { TGF } \beta \\
\text { HIF-I } \alpha \text { and HIF-2 } \alpha \\
\text { PERI }\end{array}$ & $\begin{array}{l}\text { miR-2I } \\
\text { miR-24-3P }\end{array}$ & $\begin{array}{l}\text { Proliferation, differentiation } \\
\text { Proliferation, differentiation } \\
\text { [6I] } \\
\text { [6I] } \\
\text { [62] } \\
\text { [63] }\end{array}$ \\
\hline HNSCC & miR-486-3p, miR-I0b-5p & KEGG & Proliferation, differentiation & [37] \\
\hline The oral cavity & miRNA3I & SOCSI/STAT6 & Proliferation, differentiation & [38] \\
\hline Salivary glands adenoid cystic cancer & mRNA & ERKI/2, MAPKand NGF-NTRKI & Proliferation, differentiation & [64] \\
\hline TSCC & miR-5I2-3p & Gas2 & Proliferation, differentiation & [65] \\
\hline Pancreatic cancer & mRNA & NK cells & Transfer, metastasis & [66] \\
\hline NPC & LMPI & NTRK, 2Akt and ERK & Transfer, metastasis & Transfer, metastasis \\
\hline [69] \\
[70]
\end{tabular}

Abbreviations: OSCC, oral squamous cell carcinoma; HNSCC, head and neck squamous cell carcinoma; TSCC, tongue squamous cell carcinoma; NPC, nasopharyngeal carcinoma.

\section{Acknowledgments}

This work was supported by grants from the Natural Science Foundation of Zhejiang Province (Grant Nos. LY19H160014, LY20H130001, LQ21H130001); Medical and Health Research Project of Zhejiang Province (Grant Nos. 2019ZD018, 2021KY307); Ningbo Health Branding Subject Fund (Grant No. PPXK2018-02); Ningbo Natural Science Foundation (Grant Nos. 2018A610361, 2019A610319, 202003N4239); Ningbo "Technology Innovation 2025" Major Special Project (Grant Nos. 2018B10015, 2020Z097).

\section{Disclosure}

The authors report no conflicts of interest in this work.

\section{References}

1. Pilleron S, Sarfati D, Janssen-Heijnen M, et al. Global cancer incidence in older adults, 2012 and 2035: a population-based study. Int $J$ Cancer. 2019;144(1):49-58. doi:10.1002/ijc.31664

2. Pedersen AML, Sørensen CE, Proctor GB, Carpenter GH, Ekström J. Salivary secretion in health and disease. J Oral Rehabil. 2018;45 (9):730-746. doi:10.1111/joor.12664

3. Han Y, Jia L, Zheng Y, Li W. Salivary exosomes: emerging roles in systemic disease. Int J Biol Sci. 2018;14(6):633-643. doi:10.7150/ ijbs. 25018
4. Raab-Traub N, Dittmer DP. Viral effects on the content and function of extracellular vesicles. Nat Rev Microbiol. 2017;15(9):559-572. doi:10.1038/nrmicro.2017.60

5. Johnstone RM, Adam M, Hammond JR, Orr L, Turbide C. Vesicle formation during reticulocyte maturation. Association of plasma membrane activities with released vesicles (exosomes). The Journal of Biological Chemistry. 1987;262(19):9412-9420. doi:10.1016/ S0021-9258(18)48095-7.

6. Latifkar A, Cerione RA, Antonyak MA. Probing the mechanisms of extracellular vesicle biogenesis and function in cancer. Biochem Soc Trans. 2018;46(5):1137-1146. doi:10.1042/BST20180523

7. Wu M, Ouyang Y, Wang $\mathrm{Z}$, et al. Isolation of exosomes from whole blood by integrating acoustics and microfluidics. Proc Natl Acad Sci U S A. 2017;114(40):10584-10589. doi:10.1073/ pnas. 1709210114

8. Spaull R, McPherson B, Gialeli A, et al. Exosomes populate the cerebrospinal fluid of preterm infants with post-haemorrhagic hydrocephalus. Int J Develop Neurosc. 2019;73:59-65. doi:10.1016/j. ijdevneu.2019.01.004

9. Street JM, Koritzinsky EH, Glispie DM, Yuen PST. Urine exosome isolation and characterization. Methods Mol Biol. 2017;1641:413-423.

10. Cheshmi B, Cheshomi H. Salivary exosomes: properties, medical applications, and isolation methods. Mol Biol Rep. 2020;47 (8):6295-6307. doi:10.1007/s11033-020-05659-1

11. Zheng X, Chen F, Zhang J, Zhang Q, Lin J. Exosome analysis: a promising biomarker system with special attention to saliva. J Membr Biol. 2014;247(11):1129-1136. doi:10.1007/s00232-014-9717-1

12. Fu W, Lei C, Liu S, et al. CAR exosomes derived from effector CAR$\mathrm{T}$ cells have potent antitumour effects and low toxicity. Nat Commun. 2019;10(1):4355. doi:10.1038/s41467-019-12321-3

13. Li J, Peng K, Li Y, et al. Exosome-mimetic supramolecular vesicles with reversible and controllable fusion and fission. Angew Chem Int Ed Engl. 2020;59(48):21510-21514. doi:10.1002/anie.202010257 
14. Wang J, Dong Y, Li Y, et al. Designer exosomes for active targeted chemo-photothermal synergistic tumor therapy. Adv Funct Mater. 2018;28(18):1707360. doi:10.1002/adfm.201707360

15. Nonaka T, Wong DTW. Saliva-exosomics in cancer: molecular characterization of cancer-derived exosomes in saliva. The Enzymes. 2017;42:125-151.

16. Nguyen VVT, Witwer KW, Verhaar MC, Strunk D, van Balkom BWM. Functional assays to assess the therapeutic potential of extracellular vesicles. J Extracellular Vesicles. 2020;10(1):e12033. doi:10.1002/jev2.12033

17. Ferreira AF, Calin GA, Picanço-Castro V, Kashima S, Covas DT, de Castro FA. Hematopoietic stem cells from induced pluripotent stem cells - considering the role of microRNA as a cell differentiation regulator. J Cell Sci. 2018;131(4):jcs203018. doi:10.1242/jcs.203018

18. Palanisamy V, Sharma S, Deshpande A, Zhou H, Gimzewski J, Wong DT. Nanostructural and transcriptomic analyses of human saliva derived exosomes. PLoS One. 2010;5(1):e8577. doi:10.1371/journal.pone. 0008577

19. Zlotogorski-Hurvitz A, Dayan D, Chaushu G, et al. Human salivaderived exosomes: comparing methods of isolation. J Histochem Cytochem. 2015;63(3):181-189. doi:10.1369/0022155414564219

20. Shao H, Im H, Castro CM, Breakefield X, Weissleder R, Lee H. New technologies for analysis of extracellular vesicles. Chem Rev. 2018;118(4):1917-1950. doi:10.1021/acs.chemrev.7b00534

21. Li P, Kaslan M, Lee SH, Yao J, Gao Z. Progress in exosome isolation techniques. Theranostics. 2017;7(3):789-804. doi:10.7150/thno.18133

22. Chen Y, Zhu Q, Cheng L, et al. Exosome detection via the ultrafastisolation system: EXODUS. Nat Methods. 2021;18(2):212-218. doi:10.1038/s41592-020-01034-X

23. Kim H, Lee JW, Han G, Kim K, Yang Y, Kim SH. Extracellular vesicles as potential theranostic platforms for skin diseases and aging. Pharmaceutics. 2021;13(5):760. doi:10.3390/pharmaceutics 13050760

24. Zheng X, Chen F, Zhang Q, et al. Salivary exosomal PSMA7: a promising biomarker of inflammatory bowel disease. Protein Cell. 2017;8(9):686-695. doi:10.1007/s13238-017-0413-7

25. Han P, Bartold PM, Salomon C, Ivanovski S. Salivary outer membrane vesicles and DNA methylation of small extracellular vesicles as biomarkers for periodontal status: a pilot study. Int J Mol Sci. 2021;22(5):2423. doi:10.3390/ijms22052423

26. Byun JS, Hong SH, Choi JK, Jung JK, Lee HJ. Diagnostic profiling of salivary exosomal microRNAs in oral lichen planus patients. Oral Dis. 2015;21(8):987-993. doi:10.1111/odi.12374

27. Melo SA, Luecke LB, Kahlert C, et al. Glypican-1 identifies cancer exosomes and detects early pancreatic cancer. Nature. 2015;523 (7559):177-182. doi:10.1038/nature14581

28. Tang MK, Wong AS. Exosomes: emerging biomarkers and targets for ovarian cancer. Cancer Lett. 2015;367(1):26-33. doi:10.1016/j. canlet.2015.07.014

29. Kholia S, Ranghino A, Garnieri P, et al. Extracellular vesicles as new players in angiogenesis. Vascul Pharmacol. 2016;86:64-70. doi:10.1016/j.vph.2016.03.005

30. Dong X, Bai X, Ni J, et al. Exosomes and breast cancer drug resistance. Cell Death Dis. 2020;11(11):987. doi:10.1038/s41419020-03189-z

31. Ye L, Zhang Q, Cheng Y, et al. Tumor-derived exosomal HMGB1 fosters hepatocellular carcinoma immune evasion by promoting TIM$1(+)$ regulatory B cell expansion. J Immunother Cancer. 2018;6 (1):145. doi:10.1186/s40425-018-0451-6

32. Zhou C, Wei W, Ma J, et al. Cancer-secreted exosomal miR-1468-5p promotes tumor immune escape via the immunosuppressive reprogramming of lymphatic vessels. Mol Ther. 2021;29(4):1512-1528. doi:10.1016/j.ymthe.2020.12.034

33. Pi F, Binzel DW, Lee TJ, et al. Nanoparticle orientation to control RNA loading and ligand display on extracellular vesicles for cancer regression. Nat Nanotechnol. 2018;13(1):82-89. doi:10.1038/s41565017-0012-z
34. Siravegna G, Marsoni S, Siena S, Bardelli A. Integrating liquid biopsies into the management of cancer. Nat Rev Clin Oncol. 2017;14(9):531-548. doi:10.1038/nrclinonc.2017.14

35. Nonaka T, Wong DTW. Liquid biopsy in head and neck cancer: promises and challenges. $J$ Dent Res. 2018;97(6):701-708. doi: $10.1177 / 0022034518762071$

36. Wang Y, Springer S, Mulvey CL, et al. Detection of somatic mutations and HPV in the saliva and plasma of patients with head and neck squamous cell carcinomas. Sci Transl Med. 2015;7 (293):293ra104. doi:10.1126/scitranslmed.aaa8507

37. Langevin S, Kuhnell D, Parry T, et al. Comprehensive microRNAsequencing of exosomes derived from head and neck carcinoma cells in vitro reveals common secretion profiles and potential utility as salivary biomarkers. Oncotarget. 2017;8(47):82459-82474. doi:10.18632/oncotarget.19614

38. Cai J, Qiao B, Gao N, Lin N, He W. Oral squamous cell carcinoma-derived exosomes promote M2 subtype macrophage polarization mediated by exosome-enclosed miR-29a-3p. Am J Physiol Cell Physiol. 2019;316(5):C731-c740. doi:10.1152/ ajpcell.00366.2018

39. Liu CJ, Lin SC, Yang CC, Cheng HW, Chang KW. Exploiting salivary miR-31 as a clinical biomarker of oral squamous cell carcinoma. Head Neck. 2012;34(2):219-224. doi:10.1002/hed.21713

40. Lin Y, Dong H, Deng W, et al. Evaluation of salivary exosomal chimeric GOLM1-NAA35 RNA as a potential biomarker in Esophageal carcinoma. Clin Cancer Res. 2019;25(10):3035-3045. doi:10.1158/1078-0432.CCR-18-3169

41. Zhang L, Xiao H, Karlan S, et al. Discovery and preclinical validation of salivary transcriptomic and proteomic biomarkers for the noninvasive detection of breast cancer. PLoS One. 2010;5(12):e15573. doi:10.1371/journal.pone.0015573

42. Dalla PV, Santos J, Milthorpe BK, Padula MP. Selectively-packaged proteins in breast cancer extracellular vesicles involved in metastasis. Int J Mol Sci. 2020;21(14):4990. doi:10.3390/ijms21144990

43. Lau C, Kim Y, Chia D, et al. Role of pancreatic cancer-derived exosomes in salivary biomarker development. $J$ Biol Chem. 2013;288(37):26888-26897. doi:10.1074/jbc.M113.452458

44. Sun Y, Huo C, Qiao Z, et al. Comparative proteomic analysis of exosomes and microvesicles in human saliva for lung cancer. J Proteome Res. 2018;17(3):1101-1107. doi:10.1021/acs.jproteome.7b00770

45. Sun Y, Liu S, Qiao Z, et al. Systematic comparison of exosomal proteomes from human saliva and serum for the detection of lung cancer. Anal Chim Acta. 2017;982:84-95. doi:10.1016/j.aca.2017.06.005

46. Ullah M, Kodam SP, Mu Q, Akbar A. Microbubbles versus extracellular vesicles as therapeutic cargo for targeting drug delivery. ACS Nano. 2021;15(3):3612-3620. doi:10.1021/acsnano.0c10689

47. Zhu L, Chen L. Progress in research on paclitaxel and tumor immunotherapy. Cell Mol Biol Lett. 2019;24:40. doi:10.1186/s11658-0190164-y

48. Kim MS, Haney MJ, Zhao Y, et al. Engineering macrophage-derived exosomes for targeted paclitaxel delivery to pulmonary metastases: in vitro and in vivo evaluations. Nanomedicine. 2018;14(1):195-204. doi:10.1016/j.nano.2017.09.011

49. Ma J, Zhang Y, Tang K, et al. Reversing drug resistance of soft tumorrepopulating cells by tumor cell-derived chemotherapeutic microparticles. Cell Res. 2016;26(6):713-727. doi:10.1038/cr.2016.53

50. Aslan C, Kiaie SH, Zolbanin NM, et al. Exosomes for mRNA delivery: a novel biotherapeutic strategy with hurdles and hope. BMC Biotechnol. 2021;21(1):20. doi:10.1186/s12896-021-00683-w

51. Marar C, Starich B, Wirtz D. Extracellular vesicles in immunomodulation and tumor progression. Nat Immunol. 2021;22(5):560-570. doi:10.1038/s41590-021-00899-0

52. Salazar C, Nagadia R, Pandit P, et al. A novel saliva-based microRNA biomarker panel to detect head and neck cancers. Cellular Oncol. 2014;37(5):331-338. doi:10.1007/s13402-014-0188-2 
53. Baroni S, Romero-Cordoba S, Plantamura I, et al. Exosome-mediated delivery of miR-9 induces cancer-associated fibroblast-like properties in human breast fibroblasts. Cell Death Dis. 2016;7(7):e2312. doi: $10.1038 /$ cddis. 2016.224

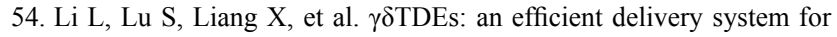
miR-138 with anti-tumoral and immunostimulatory roles on oral squamous cell carcinoma. Mol Ther Nucleic Acids. 2019;14:101113. doi:10.1016/j.omtn.2018.11.009

55. Hayashi T, Lombaert IM, Hauser BR, Patel VN, Hoffman MP. Exosomal MicroRNA transport from salivary mesenchyme regulates epithelial progenitor expansion during organogenesis. Dev Cell. 2017;40(1):95-103. doi:10.1016/j.devcel.2016.12.001

56. Xu Z, Zeng S, Gong Z, Yan Y. Exosome-based immunotherapy: a promising approach for cancer treatment. Mol Cancer. 2020;19 (1):160. doi:10.1186/s12943-020-01278-3

57. Naseri M, Bozorgmehr M, Zöller M, Ranaei Pirmardan E, Madjd Z. Tumor-derived exosomes: the next generation of promising cell-free vaccines in cancer immunotherapy. Oncoimmunology. 2020;9 (1):1779991. doi:10.1080/2162402X.2020.1779991

58. Wang M, Zhao J, Zhang L, et al. Role of tumor microenvironment in tumorigenesis. J Cancer. 2017;8(5):761-773. doi:10.7150/jca.17648

59. Jiang L, Yang W, Bian W, et al. MicroRNA-623 targets cyclin D1 to inhibit cell proliferation and enhance the chemosensitivity of cells to 5-Fluorouracil in gastric cancer. Oncol Res. 2018;27(1):19-27. doi:10.3727/096504018X15193469240508

60. Panarese I, Aquino G, Ronchi A, et al. Oral and oropharyngeal squamous cell carcinoma: prognostic and predictive parameters in the etiopathogenetic route. Expert Rev Anticancer Ther. 2019;19 (2):105-119. doi:10.1080/14737140.2019.1561288

61. Gai C, Camussi F, Broccoletti R, et al. Salivary extracellular vesicleassociated miRNAs as potential biomarkers in oral squamous cell carcinoma. BMC Cancer. 2018;18(1):439. doi:10.1186/s12885-018-4364-z

62. Li L, Li C, Wang S, et al. Exosomes derived from hypoxic oral squamous cell carcinoma cells deliver miR-21 to normoxic cells to elicit a prometastatic phenotype. Cancer Res. 2016;76(7):1770-1780. doi:10.1158/0008-5472.CAN-15-1625

63. He L, Ping F, Fan Z. Salivary exosomal miR-24-3p serves as a potential detective biomarker for oral squamous cell carcinoma screening. Biomedicine \& Pharmacotherapy = Biomedecine \& Pharmacotherapie. 2020;121:109553. doi:10.1016/j.biopha.2019.109553

64. Wang C, Niu Y, Ma C, Gong Z, Liu H. Salivary adenoid cystic carcinoma-derived exosomes promote the proliferation, migration and invasion of cancer cells. Modern Oncol. 2020;28:1067-1071.

65. Zhang L, Zhen Q, Zhan L. StudyontheeffectofexosomemiR-5123pontheproliferationoftongue squamouscellcarcinomacelllinesbydown-regulatingGas2. Chongqing Med. 2020;49:3709-3713.

66. Katsiougiannis S, Chia D, Kim Y, Singh RP, Wong DT. Saliva exosomes from pancreatic tumor-bearing mice modulate NK cell phenotype and antitumor cytotoxicity. FASEB J. 2017;31(3):9981010. doi:10.1096/fj.201600984R
67. Chen X, Li D, Huang Z, Zhong S, Cai L. Effect of exosomes derived from human Epstein-Barr virus-positive nasopharyngeal carcinoma cells on lymphangiogenesis and lymph node metastasis. Journal of Southern Medical University. 2020;40(12):1776-1783. doi:10.12122/ j.issn.1673-4254.2020.12.12.

68. Houali K, Wang X, Shimizu Y, et al. A new diagnostic marker for secreted Epstein-Barr virus encoded LMP1 and BARF1 oncoproteins in the serum and saliva of patients with nasopharyngeal carcinoma. Clin Cancer Res. 2007;13(17):4993-5000.

69. Li Z, Zhou Z, Wu X, et al. LMP1 promotes nasopharyngeal carcinoma metastasis through NTRK2-mediated anoikis resistance. Am J Cancer Res. 2020;10(7):2083-2099.

70. Wakae K, Kondo S, Pham HT, et al. EBV-LMP1 induces APOBEC3s and mitochondrial DNA hypermutation in nasopharyngeal cancer. Cancer Med. 2020;9(20):7663-7671. doi:10.1002/cam4.3357

71. Gonzalez-Begne M, Lu B, Han X, et al. Proteomic analysis of human parotid gland exosomes by multidimensional protein identification technology (MudPIT). J Proteome Res. 2009;8(3):1304-1314. doi: $10.1021 / \mathrm{pr} 800658 \mathrm{c}$

72. Carbone C, Piro G, Merz V, et al. Angiopoietin-like proteins in angiogenesis, inflammation and cancer. Int $J$ Mol Sci. 2018;19 (2):431. doi:10.3390/ijms19020431

73. Jiang K, Chen H, Fang Y, et al. Exosomal ANGPTL1 attenuates colorectal cancer liver metastasis by regulating Kupffer cell secretion pattern and impeding MMP9 induced vascular leakiness. $J$ Exp Clin Cancer Res. 2021;40(1):21. doi:10.1186/s13046-02001816-3

74. Lee BC, Kang I, Yu KR. Therapeutic features and updated clinical trials of Mesenchymal Stem Cell (MSC)-derived exosomes. J Clin Med. 2021;10(4):711. doi:10.3390/jcm10040711

75. Wei JX, Lv LH, Wan YL, et al. Vps4A functions as a tumor suppressor by regulating the secretion and uptake of exosomal microRNAs in human hepatoma cells. Hepatology (Baltimore, Md). 2015;61(4):1284-1294. doi:10.1002/hep.27660

76. Kanlikilicer P, Bayraktar R, Denizli M, et al. Exosomal miRNA confers chemo resistance via targeting Cav1/p-gp/M2-type macrophage axis in ovarian cancer. EBioMedicine. 2018;38:100-112. doi:10.1016/j.ebiom.2018.11.004

77. Xu X, Gao F, Wang J, et al. MiR-122-5p inhibits cell migration and invasion in gastric cancer by down-regulating DUSP4. Cancer Biol Ther. 2018;19(5):427-435. doi:10.1080/15384047.201 8.1423925

78. Zhang ZR, Yang N. MiR-33a-5p inhibits the growth and metastasis of melanoma cells by targeting SNAI2. Neoplasma. 2020;67(4):813824. doi:10.4149/neo_2020_190823N811

\section{Publish your work in this journal}

Cancer Management and Research is an international, peer-reviewed open access journal focusing on cancer research and the optimal use of preventative and integrated treatment interventions to achieve improved outcomes, enhanced survival and quality of life for the cancer patient.
The manuscript management system is completely online and includes a very quick and fair peer-review system, which is all easy to use. Visit http://www.dovepress.com/testimonials.php to read real quotes from published authors. 